\title{
Anisotropy in the equation of state of magnetized quark matter
}

\author{
Débora P. Menezes, ${ }^{1}$ Marcus B. Pinto, ${ }^{1}$ and Constança Providência ${ }^{2}$ \\ ${ }^{1}$ Departamento de Física, Universidade Federal de Santa Catarina Florianópolis, SC, CEP 88.040-900, Brazil \\ ${ }^{2}$ CFisUC, Department of Physics, University of Coimbra, P 3004-516 Coimbra, Portugal \\ (Received 7 April 2015; revised manuscript received 20 May 2015; published 24 June 2015)
}

\begin{abstract}
The anisotropies in the pressure obtained from the energy-momentum tensor are studied for magnetized quark matter within the su(3) Nambu-Jona-Lasinio model for both $\beta$-equilibrium matter and quark matter with equal quark chemical potentials. The effect of the magnetic field on the particle polarization, magnetization, and quark matter constituents is discussed. It is shown that the onset of the $s$ quark after chiral symmetry restoration of the $u$ and $d$ quarks gives rise to a special effect on the magnetization in the corresponding density range: A quite small magnetization just before the $s$ onset is followed by a strong increase of this quantity as soon as the $s$ quark sets in. It is also demonstrated that for $B<10^{18} \mathrm{G}$ within the two scenarios discussed, always considering a constant magnetic field, the two components of pressure are practically coincident.
\end{abstract}

DOI: 10.1103/PhysRevC.91.065205

PACS number(s): 95.30.Tg, 24.10.Jv, 21.65.Qr

The structure of the QCD phase diagram is of utmost importance in understanding many physical aspects of nature, ranging from the early universe to possible nuclear liquid-gas and hadronic quark matter phase transitions to the physics of compact objects [1]. Early analyzes performed within the Nambu-Jona-Lasinio model (NJL) framework indicate that when strongly interacting matter is subject to intense magnetic fields the QCD phase diagram boundaries are modified [2]. Some of the most important changes concern the size and location of the first-order chiral transition region since the results show that a strong magnetic field favors this type of transition. At the same time, at low temperatures, the value of the coexistence chemical potential decreases as $B$ increases in accordance with the inverse magnetic catalysis (ICM) phenomenon [3].

The low- $T$ and high- $\mu$ region where a first-order-type transition is expected to occur is currently unavailable to lattice QCD evaluations (LQCD). However, the region high- $T$ and low- $\mu$ has already been exploited using LQCD simulations which indicate, in accordance with most model predictions, that the crossover observed at $B=0$ persists when $B \neq 0$ [4-7]. On the other hand, a major disagreement between recent $L Q C D$ results $[6,7]$ and model calculations regards the dependence of crossover pseudocritical temperature, $T_{\mathrm{pc}}$, on the strength $B$ of the magnetic field. Specifically, the lattice results of Refs. [6,7], performed with $2+1$ quark flavors and physical pion mass values, predict an inverse catalysis, with $T_{\mathrm{pc}}$ decreasing with $B$, while effective models predict an increase of $T_{\mathrm{pc}}$ with $B$. This problem has been recently addressed by different groups $[8,9]$, who basically agree that the different results stem from the fact that most effective models miss back reaction effects (the indirect interaction of gluons and $B$ ) as well as the QCD asymptotic freedom phenomenon. At the same time, other important aspects of the effects of strong magnetic fields on the QCD phase diagram have already been studied, including the behavior of the coexistence chemical potential and the location of the critical end point (CEP) [10], the dependence of the CEP on strangeness, isospin, and charge asymmetry [11], and also the internal structure of the phase diagram [12].
Regarding physical observables, the understanding of magnetized quark matter is particularly important at low densities and high temperatures, which is the relevant regime for the present heavy-ion collision experiments [13], as well as at low temperatures and high densities, which is the regime concerning magnetars [14].

As far as heavy ion-collisions are concerned, the presence of a strong magnetic field most certainly plays a role despite the fact that, in principle, the field intensity should decrease very rapidly, lasting for about $1-2 \mathrm{fm} / \mathrm{c}$ only [13]. The possibility that this short time interval may [15] or may not [16] be affected by conductivity remains under dispute. The effects of strong magnetic fields and their relation with the impact parameters have also been discussed [17], while the particle yield dependence on a constant external magnetic field has been investigated in another approach [18]. Another aspect related to the presence of strong magnetic fields at the early stages of the collisions is the anisotropy of photon production in heavy-ion collisions at the RHIC energies [19]. The new PHENIX data brings some doubt on the conventional picture of thermalization and subsequent hydrodynamics or implies the possibility that a new photon production mechanism is possible. These works tell us that there is much to be done if a complete understanding of the effects of hadronic matter subject to strong magnetic fields is expected.

When we look at the recent literature on magnetars, the controversy is already present at the level of calculating the energy-momentum tensor. While some of the first works advocated that the pressure, having in mind the thermodynamical pressure obtained from the thermodynamical potential that relates pressure to density, should be isotropic [20-23], based on an interpretation given in Ref. [24], other works were based on the fact that the energy-momentum tensor gives different contributions for the parallel and perpendicular pressure [25-28] (see Ref. [29] for a discussion based on LQCD). If two different pressures are indeed present in the system, the usual way of using the equation of state as input to the Tolman-Oppenheimer-Volkoff equations (TOV) [30], which determine the structure of a spherically symmetric body of isotropic material in static gravitational equilibrium, to 
obtain compact objects macroscopic properties, as radii and masses, has to be done with care. What is normally done is to observe the value of the magnetic fields where the two pressures start to deviate and then use the TOV equations up to this strength, so that in principle the EoS used as input is practically isotropic [27,28,31]. Another important aspect is related to the contribution of the electromagnetic interaction to the pressure(s) and energy density, a term proportional to $B^{2}$, where $B$ is the magnetic field strength. Since no field larger than $10^{16} \mathrm{G}$ has been observed at the surface of a magnetar, but according to the viral theorem one could expect fields as strong as $\sim 10^{18} \mathrm{G}$ in the interior, an ad hoc exponential density-dependent magnetic field was proposed in Ref. [32] and widely adopted in subsequent works [21-23,33-41]. This ansatz, however, violates Maxwell equations. Another similar prescription for an energy-density-dependent magnetic field was proposed in Ref. [42].

To avoid the use of the TOV equations, the authors of Ref. [40] treated the anisotropic pressure as a perturbation in a way similar to the Hartle-Thorn method, generally used for slowly rotating neutron stars. In a more complete treatment, the authors consider the anisotropy in solving Einstein's field equations in axisymmetric regime with a fully general relativistic formalism [43-45]. In both cases, once the macroscopic properties are obtained, a small increase of the maximum mass is found, in contrast with the other previous works.

According to classical books on gravitation [46,47], when anisotropies are present, the concept of pressure is not so well defined. Based on the concepts discussed in these two books, in Ref. [42] a small-scale chaotic field is used and the stress tensor is modified, so that the resulting EoS is also isotropic. A curious outcome is that the increase in the maximum stellar mass is also very small, as found in Refs. [40,45]. Hence, it is clear that there is no unique way of computing magnetic field effects on compact stars.

An estimation of the maximum magnetic field intensity supported by a star before magnetic field stresses give rise to the formation of a black hole may be obtained equating the magnetic field energy of an uniform field in a sphere with the star radius $R$ to the gravitational binding energy. A maximum field of the order of $10^{18} \mathrm{G}$ is obtained in agreement with the maximum fields obtained in the framework of a relativistic magnetohydrostatic formalism, of the order of $\sim 5 \times 10^{18} \mathrm{G}$ with a nucleonic EOS [44] or $\sim 3 \times 10^{18} \mathrm{G}$ in Ref. [48] with an hyperonic EOS. It was suggested that a disordered field with $\left\langle B^{2}\right\rangle>\langle\vec{B}\rangle^{2}$ could possibly give rise to larger fields in still stable stars. The previous estimations referred to stars that are bound by gravitation. For self-bound stars larger fields could in principle exist $[25,49]$. Taking these numbers as indicative, we next consider fields $B \leqslant 1.5 \times 10^{19} \mathrm{G}$.

In the present work we investigate, within the su(3) version of the Nambu-Jona-Lasinio [50] model the quark matter polarization and magnetization, the thermodynamical pressure, and the parallel and perpendicular pressure contributions obtained from the energy-momentum tensor. We consider both $\beta$-equilibrium matter and quark matter with equal chemical potentials for the three flavors, which we call symmetric quark matter or matter with isochemical potentials throughout the text. The first scenario applies to neutron stars while the second is relevant to heavy-ion collision investigations. Some of these quantities are inputs for numerical codes that calculate the structure of neutron stars subject to strong magnetic fields.

The paper is organized as follows: In Secs. I and II, the general formalism and the resulting equation of state developed in Refs. [20,21] are revisited. In Sec. III, the expressions for the magnetization and the anisotropic pressures are shown, with some of the details given in Appendix. In Sec. IV the results are shown and discussed, and in Sec. V the final conclusions are drawn.

\section{GENERAL FORMALISM}

In order to consider both symmetric quark matter and stellar quark matter in $\beta$ equilibrium with strong magnetic fields we introduce the following Lagrangian density:

$$
\mathcal{L}=\mathcal{L}_{f}+\mathcal{L}_{l}-\frac{1}{4} F_{\mu \nu} F^{\mu \nu},
$$

which contains a quark sector, $\mathcal{L}_{f}$, a leptonic sector, $\mathcal{L}_{l}$, and the electromagnetic contribution. The quark sector is described by the su(3) version of the Nambu-Jona-Lasinio model (NJL)

$$
\mathcal{L}_{f}=\bar{\psi}_{f}\left[\gamma_{\mu}\left(i \partial^{\mu}-\hat{q}_{f} A^{\mu}\right)-\hat{m}_{c}\right] \psi_{f}+\mathcal{L}_{\text {sym }}+\mathcal{L}_{\text {det }} .
$$

The $\mathcal{L}_{\text {sym }}$ and $\mathcal{L}_{\text {det }}$ terms are given by

$$
\begin{aligned}
\mathcal{L}_{\text {sym }} & =G \sum_{a=0}^{8}\left[\left(\bar{\psi}_{f} \lambda_{a} \psi_{f}\right)^{2}+\left(\bar{\psi}_{f} i \gamma_{5} \lambda_{a} \psi_{f}\right)^{2}\right] \\
\mathcal{L}_{\operatorname{det}} & =-K\left\{\operatorname{det}_{f}\left[\bar{\psi}_{f}\left(1+\gamma_{5}\right) \psi_{f}\right]+\operatorname{det}_{f}\left[\bar{\psi}_{f}\left(1-\gamma_{5}\right) \psi_{f}\right]\right\},
\end{aligned}
$$

where $\psi_{f}=(u, d, s)^{T}$ represents a quark field with three flavors, $\hat{m}_{c}=\operatorname{diag}_{f}\left(m_{u}, m_{d}, m_{s}\right)$ with $m_{u}=m_{d} \neq m_{s}$ is the corresponding (current) mass matrix, while $\hat{q}_{f}=\operatorname{diag}\left(q_{u}, q_{d}, q_{s}\right)$ is the matrix that represents the quark electric charges. In the same equation, $\lambda_{0}=\sqrt{2 / 3} I$ where $I$ is the unit matrix in the three flavor space, and $0<\lambda_{a} \leqslant 8$ denote the Gell-Mann matrices. The t'Hooft interaction term $\left(\mathcal{L}_{\text {det }}\right)$ represents a determinant in flavor space which, for three flavors, gives a six-point interaction [51], and $\mathcal{L}_{\text {sym }}$, which is symmetric under global $U\left(N_{f}\right)_{L} \times U\left(N_{f}\right)_{R}$ transformations, corresponds to a four-point interaction in flavor space. The model is nonrenormalizable, and as a regularization scheme for the divergent ultraviolet integrals we use a sharp cutoff $\Lambda$ in three-momentum space. The parameters of the model, $\Lambda$, the coupling constants $G$ and $K$, and the current quark masses, $m_{u}$ and $m_{s}$, are determined by fitting $f_{\pi}, m_{\pi}, m_{K}$, and $m_{\xi^{\prime}}$ to their empirical values. We adopt the parametrization of the model proposed in Ref. [52]: $\Lambda=631.4 \mathrm{MeV}$, $m_{u}=m_{d}=5.5 \mathrm{MeV}, m_{s}=135.7 \mathrm{MeV}, G \Lambda^{2}=1.835$, and $K \Lambda^{5}=9.29$.

The leptonic sector is described by

$$
\mathcal{L}_{l}=\bar{\psi}_{l}\left[\gamma_{\mu}\left(i \partial^{\mu}-q_{l} A^{\mu}\right)-m_{l}\right] \psi_{l},
$$

where $l=e, \mu$. One recognizes this sector as being represented by the usual QED type of Lagrangian density. As usual, $A_{\mu}$ and $F_{\mu \nu}=\partial_{\mu} A_{\nu}-\partial_{\nu} A_{\mu}$ are used to account for the external magnetic field. We are interested in a static and constant 
magnetic field in the $z$ direction and hence we choose the gauge $A_{\mu}=\delta_{\mu 2} x_{1} B$.

\section{THE EOS}

We now need to evaluate the thermodynamical potential for the three-flavor quark sector, $\Omega_{f}$, which as usual can be written as

$$
\Omega_{f}=-P_{f}=\mathcal{E}_{f}-T \mathcal{S}_{f}-\sum_{f} \mu_{f} \rho_{f},
$$

where $P_{f}$ represents the pressure, $\mathcal{E}_{f}$ is the energy density, $T$ is the temperature, $\mathcal{S}_{f}$ is the entropy density, $\mu_{f}$ is the chemical potential, and $\rho_{f}$ is the quark number density. A similar expression can be written for the leptonic sector.

The total pressure for three flavors in $\beta$ equilibrium is then given by

$$
P\left(\mu_{f}, \mu_{l}, B\right)=\left.P_{f}\right|_{M_{f}}+\left.P_{l}\right|_{m_{l}} \pm \frac{B^{2}}{2}
$$

where our notation means that $P_{f}$ is evaluated in terms of the quark effective mass, $M_{f}$, which is determined in a (nonperturbative) self-consistent way, while $P_{l}$ is evaluated at the leptonic bare mass, $m_{l}$. The term $B^{2} / 2$ arises due to the kinetic term of the electromagnetic field, $F_{\mu \nu} F^{\mu \nu} / 4$, in the original Lagrangian density. Within the formalism used in the present work, the sign of this term comes from the stress tensor and is shown in the section where we discuss anisotropy. In the sequel our results will be presented in terms of (vacuum) subtracted pressures $(\Delta P)$ such that $P_{f}=0$ at $\mu_{f}=0(f=u, s, d)$ and $P_{l}=0$ at $\mu_{l}=0(l=e, \mu)$. With this normalization choice only the magnetic pressure, $\left( \pm B^{2} / 2\right)$, survives at vanishing chemical potentials. Again, a similar expression can be written for the leptonic sector, apart from the color index. The lepton masses are $m_{e}=0.511 \mathrm{MeV}$ and $m_{\mu}=105.66 \mathrm{MeV}$.

In the mean field approximation the pressure can be written as

$$
P_{f}=\theta_{u}+\theta_{d}+\theta_{s}-2 G\left(\phi_{u}^{2}+\phi_{d}^{2}+\phi_{s}^{2}\right)+4 K \phi_{u} \phi_{d} \phi_{s},
$$

where the free gas type of term is

$$
\theta_{f}=-\frac{i}{2} \operatorname{tr} \int \frac{d^{4} p}{(2 \pi)^{4}} \ln \left(-p^{2}+M_{f}^{2}\right)
$$

while the scalar condensates, $\phi_{f}$ are given by

$$
\phi_{f}=\left\langle\bar{\psi}_{f} \psi_{f}\right\rangle=-i \int \frac{d^{4} p}{(2 \pi)^{4}} \operatorname{tr} \frac{1}{\left(\not p-M_{f}+i \epsilon\right)} .
$$

According to standard Feynman rules for this model, all the traces are to be taken over color $\left(N_{c}=3\right)$ and Dirac space, but not flavor.

The effective quark masses can be obtained selfconsistently from

$$
M_{i}=m_{i}-4 G \phi_{i}+2 K \phi_{j} \phi_{k}
$$

with $(i, j, k)$ being any permutation of $(u, d, s)$. So, to determine the EOS for the su(3) NJL at finite density and in the presence of a magnetic field we need to know the condensates, $\phi_{f}$, as well as the contribution from the gas of quasiparticles, $\theta_{f}$. Both quantities, which are related by $\phi_{f} \sim d \theta_{f} / d M_{f}$, have been evaluated with great detail in Refs. [20,21] so that here we just quote the results:

$$
\theta_{f}=\left(\theta_{f}^{\mathrm{vac}}+\theta_{f}^{\mathrm{mag}}+\theta_{f}^{\mathrm{med}}\right)_{M_{f}},
$$

where the vacuum contribution reads

$$
\theta_{f}^{\mathrm{vac}}=-\frac{N_{c}}{8 \pi^{2}}\left\{M_{f}^{4} \ln \left[\frac{\left(\Lambda+\epsilon_{\Lambda}\right)}{M_{f}}\right]-\epsilon_{\Lambda} \Lambda\left(\Lambda^{2}+\epsilon_{\Lambda}^{2}\right)\right\},
$$

and where we have also defined $\epsilon_{\Lambda}=\sqrt{\Lambda^{2}+M_{f}^{2}}$ with $\Lambda$ representing a noncovariant ultraviolet cutoff, the finite magnetic contribution is given by

$$
\theta_{f}^{\mathrm{mag}}=\frac{N_{c}\left(\left|q_{f}\right| B\right)^{2}}{2 \pi^{2}}\left[\zeta^{\prime}\left(-1, x_{f}\right)-\frac{1}{2}\left(x_{f}^{2}-x_{f}\right) \ln x_{f}+\frac{x_{f}^{2}}{4}\right],
$$

with $\quad x_{f}=M_{f}^{2} /\left(2\left|q_{f}\right| B\right) \quad$ while $\quad \zeta^{\prime}\left(-1, x_{f}\right)=$ $d \zeta\left(z, x_{f}\right) /\left.d z\right|_{z=-1}$ where $\zeta\left(z, x_{f}\right)$ is the Riemann-Hurwitz $\zeta$ function. To take further derivatives, as well as for numerical purposes, it is useful to use the following representation for this quantity:

$$
\zeta^{\prime}\left(-1, x_{f}\right)=\zeta^{\prime}(-1,0)+\frac{x_{f}}{2}\left[x_{f}-1-\ln (2 \pi)+\psi^{(-2)}\left(x_{f}\right)\right],
$$

where $\psi^{(m)}\left(x_{f}\right)$ is the $m$ th polygamma function and the $x_{f}$ independent constant is $\zeta^{\prime}(-1,0)=-1 / 12$. The medium contribution can be written as

$$
\begin{aligned}
\theta_{f}^{\mathrm{med}}= & T \sum_{k=0}^{k_{f, \max }} \alpha_{k} \frac{\left|q_{f}\right| B N_{c}}{4 \pi^{2}} \\
& \times \int_{-\infty}^{+\infty} d p\left[\ln \left(1+\exp \left[-\left(E_{f}^{*}-\mu_{f}\right) / T\right]\right)\right. \\
& \left.+\ln \left(1+\exp \left[-\left(E_{f}^{*}+\mu_{f}\right) / T\right]\right)\right],
\end{aligned}
$$

with $\alpha_{0}=1, \alpha_{k>0}=2$. In the above equation we have defined the energy dispersion

$$
E_{f}^{*}=\sqrt{p^{2}+s_{f}(k, B)^{2}}, \quad s_{f}(k, B)=\sqrt{M_{f}^{2}+2\left|q_{f}\right| B k} .
$$

When considering just the zero-temperature case, Eq. (2.11) becomes

$$
\begin{aligned}
\theta_{f}^{\mathrm{med}}= & \sum_{k=0}^{k_{f, \max }} \alpha_{k} \frac{\left|q_{f}\right| B N_{c}}{4 \pi^{2}}\left[\mu_{f} \sqrt{\mu_{f}^{2}-s_{f}(k, B)^{2}}-s_{f}(k, B)^{2}\right. \\
& \left.\times \ln \left(\frac{\mu_{f}+\sqrt{\mu_{f}^{2}-s_{f}(k, B)^{2}}}{s_{f}(k, B)}\right)\right]
\end{aligned}
$$


where $s_{f}(k, B)=\sqrt{M_{f}^{2}+2\left|q_{f}\right| B k}$. At $T=0$, the upper Landau level (or the nearest integer) is defined by

$$
k_{f, \max }=\frac{\mu_{f}^{2}-M_{f}^{2}}{2\left|q_{f}\right| B}=\frac{p_{f, F}^{2}}{2\left|q_{f}\right| B} .
$$

The condensates $\phi_{f}$ entering the quark pressure at finite density and in the presence of an external magnetic field can be written as

$$
\phi_{f}=\left(\phi_{f}^{\mathrm{vac}}+\phi_{f}^{\mathrm{mag}}+\phi_{f}^{\mathrm{med}}\right)_{M_{f}}
$$

where

$$
\begin{aligned}
\phi_{f}^{\mathrm{vac}}= & -\frac{M_{f} N_{c}}{2 \pi^{2}}\left[\Lambda \epsilon_{\Lambda}-M_{f}^{2} \ln \left(\frac{\Lambda+\epsilon_{\Lambda}}{M_{f}}\right)\right] \\
\phi_{f}^{\mathrm{mag}}= & -\frac{M_{f}\left|q_{f}\right| B N_{c}}{2 \pi^{2}}\left[\ln \Gamma\left(x_{f}\right)-\frac{1}{2} \ln (2 \pi)+x_{f}\right. \\
& \left.-\frac{1}{2}\left(2 x_{f}-1\right) \ln \left(x_{f}\right)\right]
\end{aligned}
$$

and

$$
\phi_{f}^{\mathrm{med}}=\sum_{k=0}^{k_{f, \max }} \alpha_{k} \frac{M_{f}\left|q_{f}\right| B N_{c}}{4 \pi^{2}} \int_{-\infty}^{+\infty} d p \frac{\left(f_{+}+f_{-}\right)}{E_{f}^{*}},
$$

where the Fermi distribution functions are

$$
f_{ \pm}=1 /\left\{1+\exp \left[\left(E_{f}^{*} \mp \mu_{f}\right) / T\right]\right\} .
$$

The quark density reads

$$
\rho_{f}=\sum_{k=0}^{k_{f, \max }} \alpha_{k} \frac{\left|q_{f}\right| B N_{c}}{2 \pi^{2}} \int_{-\infty}^{+\infty} d p\left(f_{+}-f_{-}\right) .
$$

At $T=0$, Eqs. (2.17) and (2.19) become

$$
\begin{aligned}
\phi_{f}^{\mathrm{med}}= & \sum_{k=0}^{k_{f, \max }} \alpha_{k} \frac{M_{f}\left|q_{f}\right| B N_{c}}{2 \pi^{2}} \\
& \times\left[\ln \left(\frac{\mu_{f}+\sqrt{\mu_{f}^{2}-s_{f}(k, B)^{2}}}{s_{f}(k, B)}\right)\right],
\end{aligned}
$$

and

$$
\rho_{f}=\sum_{k=0}^{k_{f, \max }} \alpha_{k} \frac{\left|q_{f}\right| B N_{c}}{2 \pi^{2}} k_{F, f},
$$

where $k_{F, f}=\sqrt{\mu_{f}^{2}-s_{f}(k, B)^{2}}$. The entropy density $\mathcal{S}_{f}=$ $-(\partial \Omega / \partial T)$ is

$$
\begin{aligned}
\mathcal{S}_{f}= & -\sum_{f} \sum_{k=0}^{k_{f, \max }} \alpha_{k} \frac{\left|q_{f}\right| B N_{c}}{4 \pi^{2}} \int_{-\infty}^{+\infty} d p\left[f_{+} \ln \left(f_{+}\right)+\left(1-f_{+}\right)\right. \\
& \left.\times \ln \left(1-f_{+}\right)+\left(f_{+} \leftrightarrow f_{-}\right)\right] .
\end{aligned}
$$

The corresponding leptonic contributions can be trivially obtained from the above quantities by replacing $M_{f} \rightarrow m_{l}$, $\left|q_{f}\right| \rightarrow\left|q_{l}\right|$, and $\mu_{f} \rightarrow \mu_{l}$. Since the leptonic masses are unaffected by strong interactions, one considers their bare values which do not depend on $T, \mu$, and $B$ as opposed to the effective $M_{f}$ masses. Therefore, the only piece which effectively contributes to the subtracted pressure, defined as $\Delta P=P(T, \mu, B)-P(0,0, B)$, is

$$
\begin{aligned}
P_{l}^{\text {med }}= & T \sum_{k=0}^{k_{f, \max }} \alpha_{k} \frac{\left|q_{l}\right| B}{4 \pi^{2}} \\
& \times \int_{-\infty}^{+\infty} d p\left[\ln \left(1+\exp \left[-\left(E_{l}-\mu_{l}\right) / T\right]\right)\right. \\
& \left.+\ln \left(1+\exp \left[-\left(E_{l}+\mu_{l}\right) / T\right]\right)\right],
\end{aligned}
$$

from which the leptonic density can be written as

$$
\rho_{l}=\sum_{k=0}^{k_{f, \max }} \alpha_{k} \frac{\left|q_{l}\right| B}{2 \pi^{2}} \int_{-\infty}^{+\infty} d p\left(l_{+}-l_{-}\right),
$$

where $\mu_{l}$ represents the leptonic chemical potential. The quantities $E_{l}$ and $l_{ \pm}$can be obtained from their quark counterparts by the replacements already mentioned.

At vanishing temperatures the above expressions become

$$
\begin{aligned}
P_{l}^{\text {med }}= & \sum_{l=e}^{\mu} \sum_{k=0}^{k_{l, \max }} \alpha_{k} \frac{\left|q_{l}\right| B}{4 \pi^{2}}\left[\mu_{l} \sqrt{\mu_{l}^{2}-s_{l}(k, B)^{2}}-s_{l}(k, B)^{2}\right. \\
& \left.\times \ln \left(\frac{\mu_{l}+\sqrt{\mu_{l}^{2}-s_{l}(k, B)^{2}}}{s_{l}(k, B)}\right)\right]
\end{aligned}
$$

and

$$
\rho_{l}=\sum_{k=0}^{k_{l, \max }} \alpha_{k} \frac{\left|q_{l}\right| B}{2 \pi^{2}} k_{F, l}\left(k, s_{l}\right),
$$

where $k_{F, l}\left(k, s_{l}\right)=\sqrt{\mu_{l}^{2}-s_{l}(k, B)^{2}}$.

\section{THE ANISOTROPY IN THE PRESSURE}

The parallel and the perpendicular components of the pressure can be written in terms of the magnetization, $\mathcal{M}=$ $\partial \Delta P / \partial B$, as [24,31,37]

$$
P_{\|}=\Delta P-\frac{B^{2}}{2} \quad \text { and } \quad P_{\perp}=\Delta P-\mathcal{M} B+\frac{B^{2}}{2},
$$

where $\Delta P$ stands for the already defined subtracted pressure. For a magnetic field in the $z$ direction, the stress tensor has the form $\operatorname{diag}\left(B^{2} / 2, B^{2} / 2,-B^{2} / 2\right)$ and this explains the difference in sign appearing in the parallel and perpendicular pressures. For the leptonic sector one easily gets

$$
\begin{aligned}
\frac{d P_{l}^{\text {med }}}{d B}= & \frac{P_{l}^{\text {med }}}{B}-\frac{\left|q_{l}\right| B}{4 \pi^{2}} \sum_{k=0}^{k_{f, \max }} \alpha_{k}\left(k\left|q_{l}\right|\right) \\
& \times \int_{-\infty}^{+\infty} d p \frac{1}{E_{l}}\left[l_{+}+l_{-}\right]
\end{aligned}
$$

which, at $T=0$ becomes:

$$
\frac{d P_{l}^{\text {med }}}{d B}=\frac{P_{l}^{\text {med }}}{B}-\frac{B\left|q_{l}\right|}{2 \pi^{2}} \sum_{k=0}^{k_{\max }} \alpha_{k} \ln \left(\frac{\mu_{l}+\sqrt{\mu_{l}^{2}-s_{l}^{2}}}{s_{f}}\right)\left(k\left|q_{l}\right|\right),
$$


whereas for the quark sector one obtains

$$
\begin{aligned}
\frac{d P_{f}}{d B}= & \theta_{u}^{\prime}+\theta_{d}^{\prime}+\theta_{s}^{\prime}-4 G\left(\phi_{u} \phi_{u}^{\prime}+\phi_{d} \phi_{d}^{\prime}+\phi_{s} \phi_{s}^{\prime}\right) \\
& +4 K\left(\phi_{u}^{\prime} \phi_{d} \phi_{s}+\phi_{u} \phi_{d}^{\prime} \phi_{s}+\phi_{u} \phi_{d} \phi_{s}^{\prime}\right)
\end{aligned}
$$

where

$$
\theta_{f}^{\prime}=\left(\theta_{f}^{\prime \mathrm{vac}}+\theta_{f}^{\prime \mathrm{mag}}+\theta_{f}^{\prime \text { med }}\right)_{M_{f}}
$$

and

$$
\phi_{f}^{\prime}=\left(\phi_{f}^{\prime \text { vac }}+\phi_{f}^{\prime \text { mag }}+\phi_{f}^{\prime \text { med }}\right)_{M_{f}} .
$$

The primes denote derivatives with respect to $B$ and the explicit form of each term can be found in the appendix.

\section{RESULTS AND DISCUSSION}

In this section we present and discuss results concerning several properties of $\beta$-equilibrium quark matter and symmetric quark matter (with equal chemical potentials). We first discuss some general properties of quark matter, in particular, its particle content and particle polarization. We then investigate how the magnetization of quark matter changes with the magnetic field intensity and finally we discuss the parallel and perpendicular pressure contributions obtained from the energy-momentum tensor.

The quark spin polarization $\Delta$ is usually defined as

$$
\Delta_{i}=\frac{\rho_{i}(\uparrow)-\rho_{i}(\downarrow)}{\rho_{i}(\uparrow)+\rho_{i}(\downarrow)}, \quad i=u, d, s .
$$

In Fig. 1 it is shown how the magnetic field affects $\beta$-equilibrium matter (left panels) and symmetric quark matter (right panels), in particular, the quark and electron polarizations, their densities, and onset of total polarization. The $\beta$-equilibrium matter onset of the $s$ quark occurs just below $0.7 \mathrm{fm}^{-3}$, see Fig. 1(c), and, therefore, close to these densities the $s$ quark density is small and feels strongly the magnetic field, with total polarization being attained with $B<10^{18} \mathrm{G}$. In denser matter, the larger densities of $s$ quarks require larger magnetic field intensities for a total polarization. In symmetric quark matter the $s$ quarks set in above $0.9 \mathrm{fm}^{-3}$, a density that presently is not attained in the laboratory. In $\beta$-equilibrium matter $u$ and $d$ quarks are not totally polarized for fields below $10^{19} \mathrm{G}$; however, in symmetric quark matter $u$ and $d$ quark total polarization occurs at small densities that do not exist in quark stellar matter with a surface baryonic density which is of the order of $0.3 \mathrm{fm}^{-3}$. In Fig. 1(a) we also show information on the electron polarization. The magnetic field increases the electron content and for $10^{18} \mathrm{G}$ their density is practically constant and equal to $\sim 0.01 \mathrm{fm}^{-3}$; see Fig. 1(c). At the onset of the $s$ quark the density of electrons has always a maximum, above which the electron fraction decreases. This means that electrons are totally polarized for fields $B \gtrsim 9 \times 10^{17} \mathrm{G}$.

For totally polarized matter, all particles lie on the lowest Landau level (LLL). In this case, the dependence of the pressure on the magnetic field intensity of a gas of free particles occurs only through a multiplicative factor that defines the LLL degeneracy, and the magnetization is independent of $B$. For the NJL model the interaction terms give a nonlinear dependence to the pressure even above for totally polarized matter. This contribution becomes small when the chiral symmetry is restored. A comparison with the polarization obtained in quark matter described by the Massachusetts Institute of Technology (MIT) bag model is shown in Ref. [53], where it is also seen that the polarization of the system increases with the increase of the magnetic field and the total polarization occurs for $B \simeq 2 \times 10^{19} \mathrm{G}$ for matter in $\beta$ equilibrium.

In order to determine the two pressure contributions, the magnetization is obtained using Eq. (A1). In Fig. 2 this quantity is plotted for $\beta$-equilibrium matter, Figs. 2(a) and 2(c), and quark matter with equal chemical potentials, Figs. 2(b) and 2(d). For each case we have considered a set of densities of interest: (a) the surface baryonic density of a quark star is $\sim 2 \rho_{0}$ and in the interior we may have densities larger than $5 \rho_{0}$; (b) in heavy-ion collisions we may have densities below $\rho_{0}$ and do not expect densities as large as $5 \rho_{0}$. The magnetization has a term that explodes whenever $\mu_{f}=s_{f}$, i.e., whenever $B$ approaches a $n \neq 0$ Landau level from below; see Eq. (A7) and the corresponding discussion in Ref. [26]. The spikes in this figure occur precisely at this values of $B$. A small number of spikes occurs if only a few LL are occupied, e.g., if the magnetic field is very strong with respect to the Fermi momentum of the particle. In Figs. 2(a) and 2(b) for the densities represented this occurs for the larger fields. For the symmetric matter at smaller densities, fewer spikes are obtained for larger magnetic fields. It is interesting to notice that for $\beta$-equilibrium matter there is a range of densities between 0.5 and $0.7 \mathrm{fm}^{-3}$ where for all the fields shown the number of oscillations is small: This occurs before the onset of the $s$ quark and after the $u$ and $q$ quark restoration of chiral symmetry. In Fig. 2(c), for densities lower than $0.3 \mathrm{fm}^{-3}$ and in between 0.8 and $1.1 \mathrm{fm}^{-3}$, one can clearly sees that oscillations with high frequencies are modulated by smaller frequency oscillations. The same is seen in Fig. 2(d), for densities larger than $0.3 \mathrm{fm}^{-3}$. This superposition of fluctuations with different frequencies is due to a mixing of particles with different charges and masses. In $\beta$-equilibrium matter the oscillations defined by the $u$ quark are wider apart because the $u$ quark density is smaller and its charge larger, both effects adding to a reduction of the number of occupied LL; see Fig. 2(c). This same effect is also present in Fig. 2(d) where, for similar $u$ and $d$ quark densities, the charge of the $u$ explains the difference. Our results differ substantially from the ones obtained in Refs. [26,53] (apart from the choice of units), since the MIT bag model, used in the calculation of the quark magnetization in these references, does not present negative magnetization. Physically, this means that while the NJL model studied here presents paramagnetic $(\mathcal{M}>0)$ as well as diamagnetic $(\mathcal{M}<0)$ behavior, the MIT model, studied in Ref. [31], displays only a paramagnetic phase. This difference can be better understood by recalling that the NJL magnetization receives contributions from $\theta_{f}^{\prime \text { med }}$ and $\phi_{f}^{\prime \text { med }}$ while only the former contributes to the MIT model [31]. As remarked earlier $\theta_{f}$ represents the contribution from a gas of quasiparticles and $\phi_{f}$ represents the quark condensate. As Eq. (A7) reveals, $\phi_{f}^{\text {med }}$ may present divergencies leading to the oscillation between diamagnetic and paramagnetic states observed in our results (before the LLL is reached). 


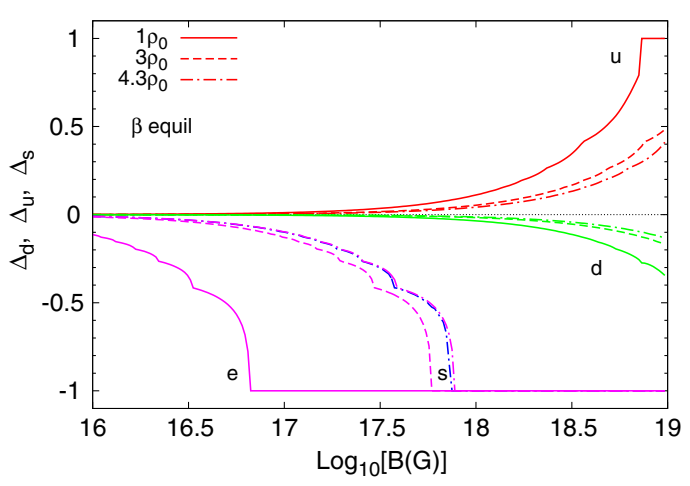

(a)

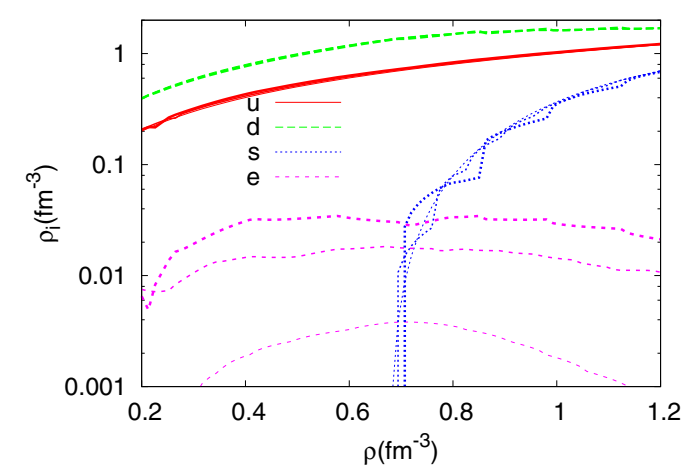

(c)

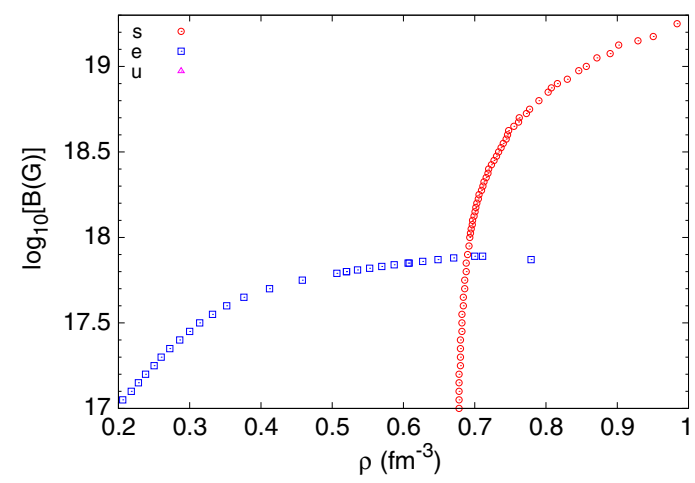

(e)

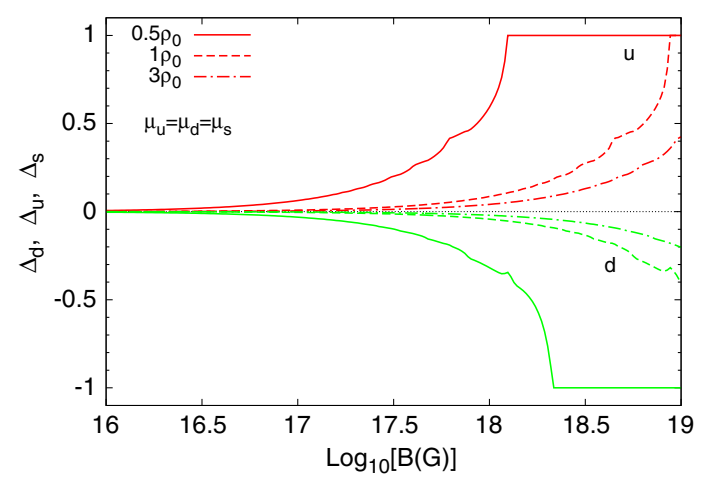

(b)

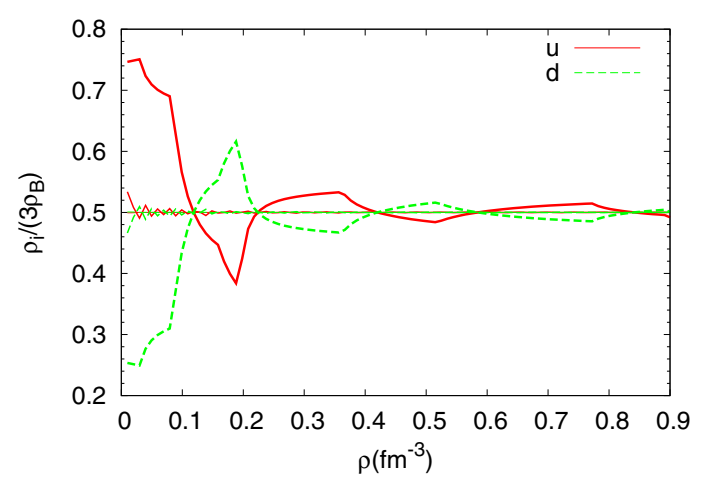

(d)

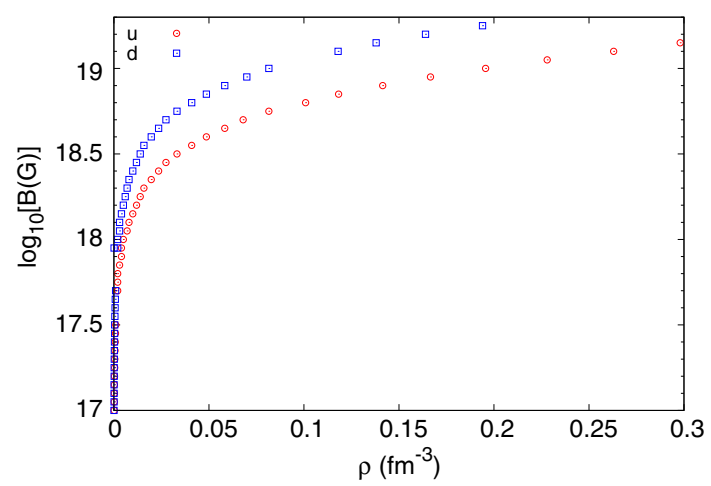

(f)

FIG. 1. (Color online) $\beta$-equilibrium quark matter (left panels) and symmetric quark matter (right panels): (a) and (b) particle polarizations for several densities defined in terms of the saturation density $\rho_{0}=0.16 \mathrm{fm}^{-3}$, (c) and (d) particle densities, absolute in panel (c) and relative in panel (d), for $10^{17}, 10^{18}$, and $10^{19} \mathrm{G}$ magnetic field intensities, respectively, from the thinner to the thicker lines; (e) total polarization onset density for the $s$ quark and the electron in $\beta$-equilibrium matter, and (f) for the $u$ and $d$ quarks in symmetric matter. For the densities representing $u$ and $d$ quarks, the total polarization occurs for stronger fields and in symmetric matter the $s$ quark sets in at larger densities.

In Fig. 3 the two pressure contributions defined in Eq. (3.1), except for the terms proportional to $B^{2}$, are plotted for both $\beta$-equilibrium matter (left panels) and symmetric matter (right panels). As in the previous discussion for each scenario we take the same representative values of the magnetic field intensity or baryonic density. One should notice that when we analyze matter in $\beta$ equilibrium, we have in mind that quark stars have no crust, since the pressure is zero for a still finite density, as clearly seen in Fig. 3(a). One of the effects of the magnetic field could be a change of the density at the surface, although nonnegligible effects occur only for magnetic fields above $10^{18} \mathrm{G}$.
A mantle could have been added as was done in Ref. [54]. Moreover, in Ref. [55] it was shown that within the NJL a much larger electron chemical potential is expected at the surface, allowing more easily for a mantle to exist. Understanding how this mantle could be affected in the presence of a magnetic field is an interesting question that, however, is beyond the scope of the present work.

For fields $B<10^{18} \mathrm{G}$ both contributions, the parallel and the perpendicular pressures, are practically coincident. However, due to the magnetization contribution entering the perpendicular pressure, discontinuities occur whenever a new 


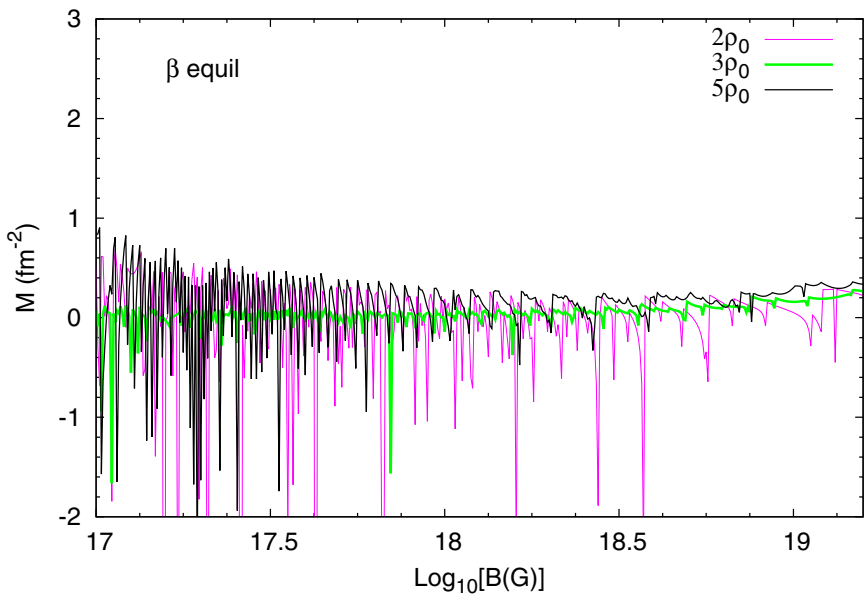

(a)

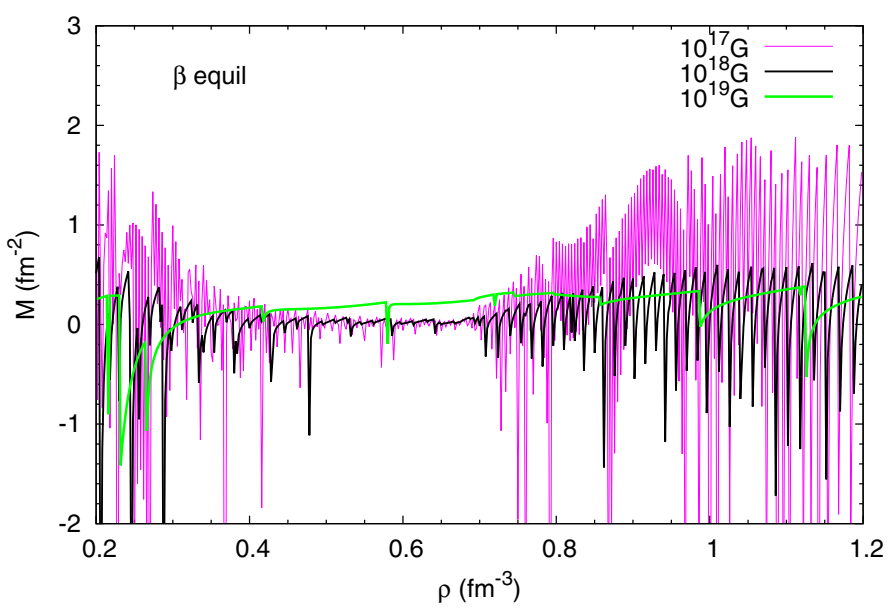

(c)

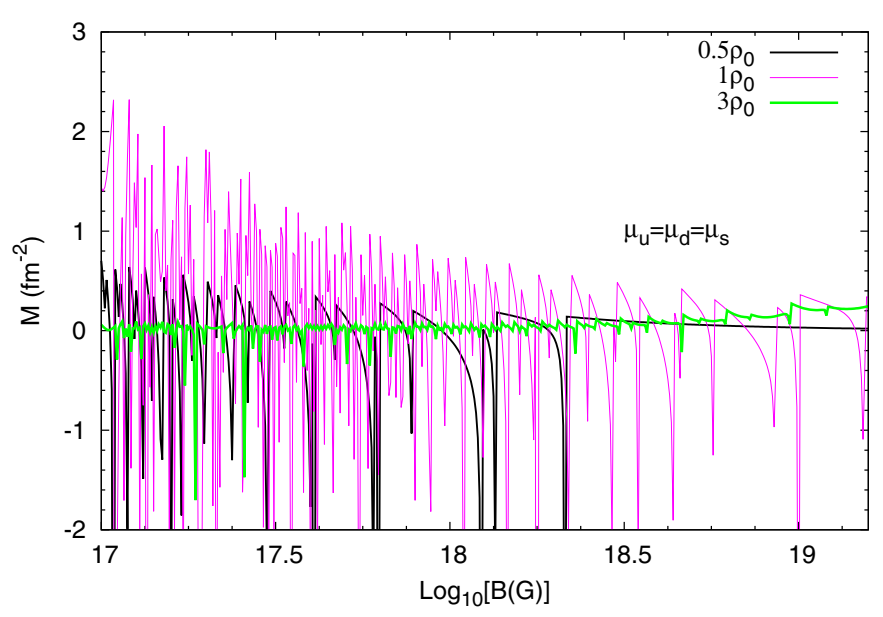

(b)

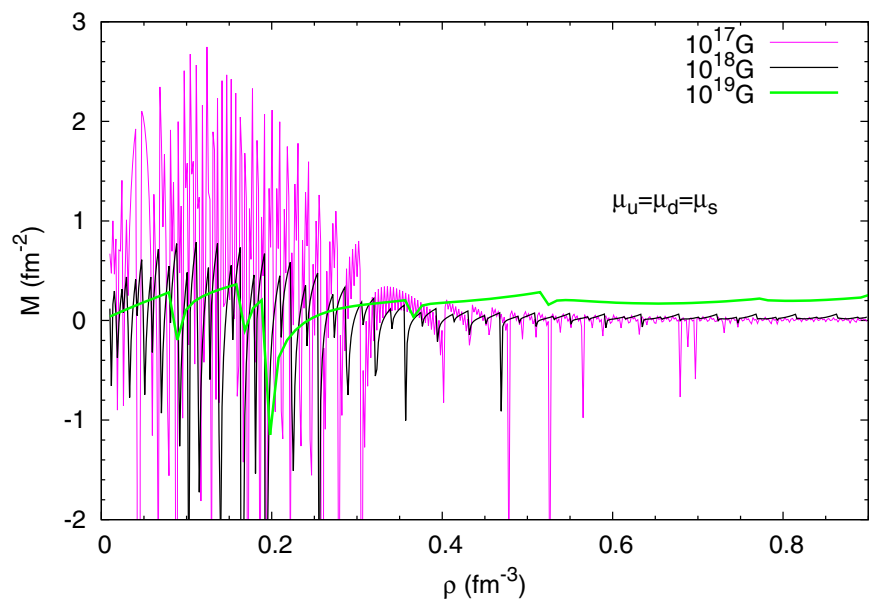

(d)

FIG. 2. (Color online) Magnetization for (a) and (c) $\beta$-equilibrium matter and (b) and (d) isochemical potential quark matter. Densities of interest for each scenario are considered. In panels (a) and (b) the magnetization is shown as a function of $B$ and in panels (c) and (d) as a function of the baryonic density.

LL level is reached, giving rise to a series of discontinuities that would correspond to unstable regions, since $d \mathcal{M} / d B<0$. Let us point out that in the region $0.5<\rho<0.7 \mathrm{fm}^{-3}$, and for $B<10^{18} \mathrm{G}$, these discontinuous contributions to the perpendicular pressure contribution are practically zero, because, as seen before, the magnetization is very small in this range of densities. As compared with calculations performed with the MIT bag model [31], the behavior is the same, except that the deviation of the two pressures takes place at even larger magnetic fields with the MIT bag model. Nevertheless, had we considered not only the matter contribution but also the contributions from the the terms proportional to $B^{2}$, the deviation would start at lower magnetic field intensities. The exact value of the magnetic field where both pressures start to deviate depends on the model used and on the chosen fixed density (or corresponding chemical potential), as can be seen when we compare our results with the ones shown in Refs. [26,53], but the qualitative results in average are the same.

One should bear in mind that the discontinuities seen in Fig. 3 are washed out at finite temperatures as the ones that we expect in heavy-ion collisions [56]. As suggested in Refs. [24,26,49] the unstable regions may give rise to domains with disordered fields that would allow continuous phase transitions between regions with a different number of LL.

Finally, we plot in Fig. 4, top figures, the energy density as a function of the number density, and in the figures at the bottom, a quark binding energy for the cases of matter in $\beta$ equilibrium (left panels) and isochemical potential matter (right panels). The energy density does not depend on the strength of the magnetic field, what corroborates the fact that thermodynamic consistency is achieved [26]. On the other hand, as already expected from previously published results $[20,21]$, the density per particle decreases with the increase of the magnetic field due to the change of the quark density with the magnetic field.

\section{FINAL REMARKS}

In this work we have examined the effects of strong magnetic fields in quark matter, as described by the NJL model, 


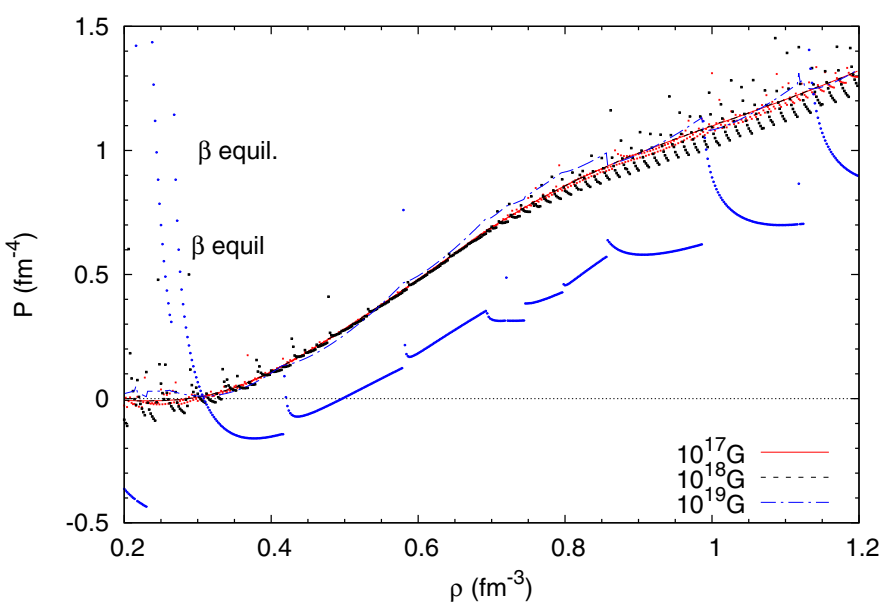

(a)

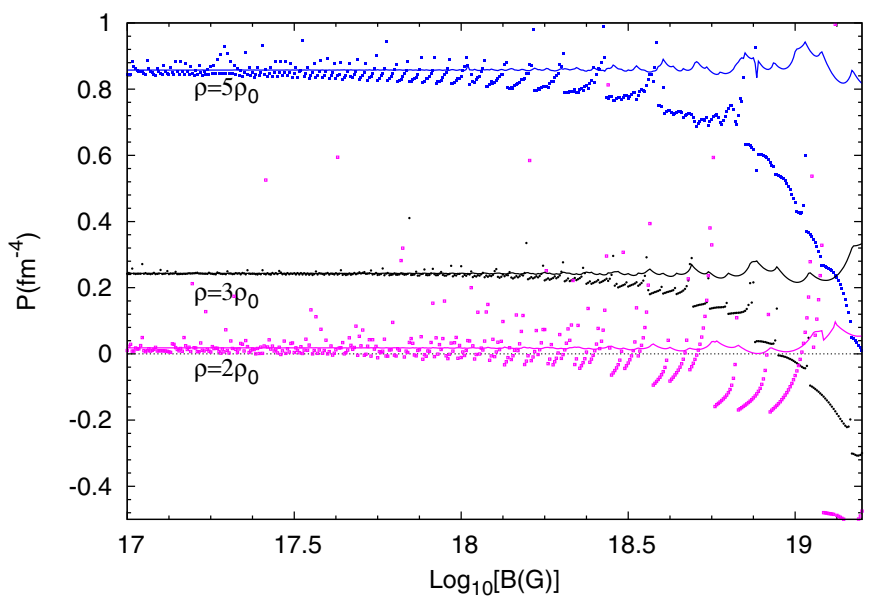

(c)

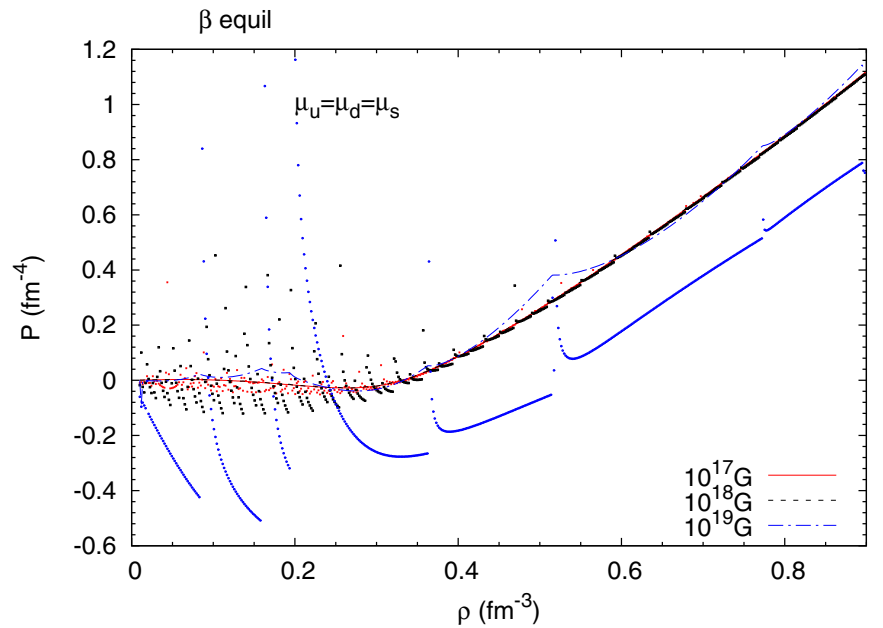

(b)

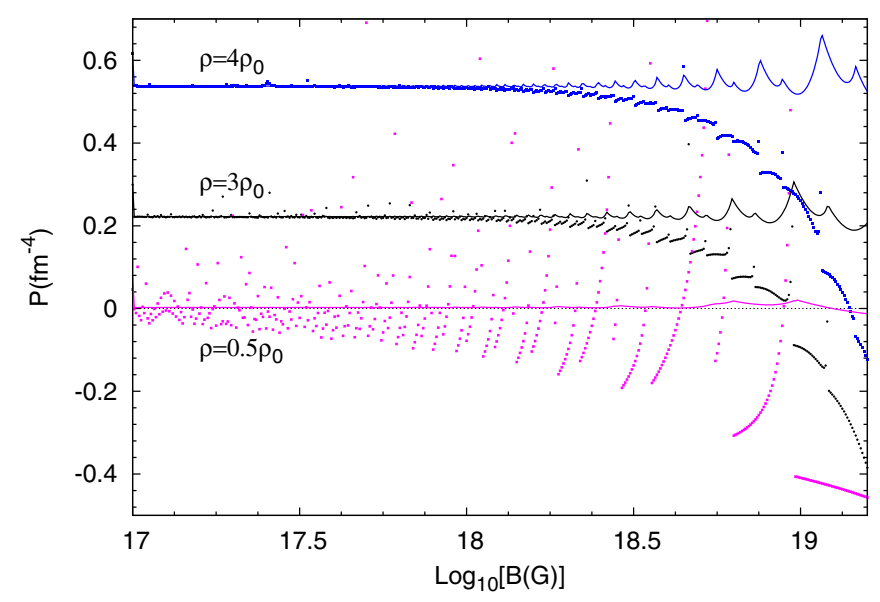

(d)

FIG. 3. (Color online) Parallel (lines) and perpendicular (marks) pressures for (a) and (c) $\beta$-equilibrium matter and (b) and (d) isochemical potential quark matter. Densities of interest for each scenario are considered. In panels (a) and (b) the pressures are shown as a function of the baryonic density and in panels (c) and (d) as a function of $B$. For fields $B \leqslant 10^{18} \mathrm{G}$ the perpendicular press falls on top of the parallel contribution except for the van Alphen oscillations due to the magnetization contribution.

paying special attention to effects due to pressure anisotropy. Two scenarios were investigated: matter in $\beta$ equilibrium, as the one possibly present in quark stars or in the core of hybrid stars, and symmetric matter with isochemical potentials, as the one present in heavy-ion collisions. For the first case, large densities and magnetic fields up to the order of $10^{18} \mathrm{G}$ are of interest, whereas for symmetric matter, the relevant densities are somewhat smaller while the magnetic fields can be larger.

Part of our work has been devoted to the analysis of quark matter constituents, the onset of $s$ quarks, and how much polarized matter can be present when different field intensities are considered since these are important ingredients for stellar modeling.

Magnetization, which is an important quantity for the description of magnetized matter, has also been considered in the present work and by investigating this quantity we conclude that the amount of spikes, related to the filling of the LL, depends quite substantially on the density and on the scenario examined. Of particular interest is the fact that very few spikes are seen for densities between 0.5 and $0.7 \mathrm{fm}^{-3}$ in $\beta$-equilibrium matter, no matter the intensity of the magnetic field, because the particles that constitute matter occupy only a few LL. For symmetric matter, the same pattern is found for densities larger than $0.5 \mathrm{fm}^{-3}$, when the number of occupied LL are small for the three different quarks.

This effect in $\beta$-equilibrium matter is due to the late onset of the $s$ quark, precisely for $\rho \gtrsim 0.7 \mathrm{fm}^{-3}$. Due to its large constituent mass magnetic fields satisfying $B \lesssim 10^{18} \mathrm{G}$, as the ones possibly existing inside magnetars, give rise to the filling of many LL and large contributions to the magnetization and perpendicular pressure, in contrast to the behavior at densities just below the $s$ quark onset. This effect is clearly seen by comparing the pressure for $\rho=3 \rho_{0}$ corresponding to $u$ and $d$ quark matter with restored chiral symmetry with the pressure obtained for either $\rho=2 \rho_{0}$ or $\rho=5 \rho_{0}$ as a function of $B$ : In the first case chiral symmetry is still not completely restored and in the second the onset of $s$ quark has occurred. If a quark model with complete chiral symmetry restored, such 


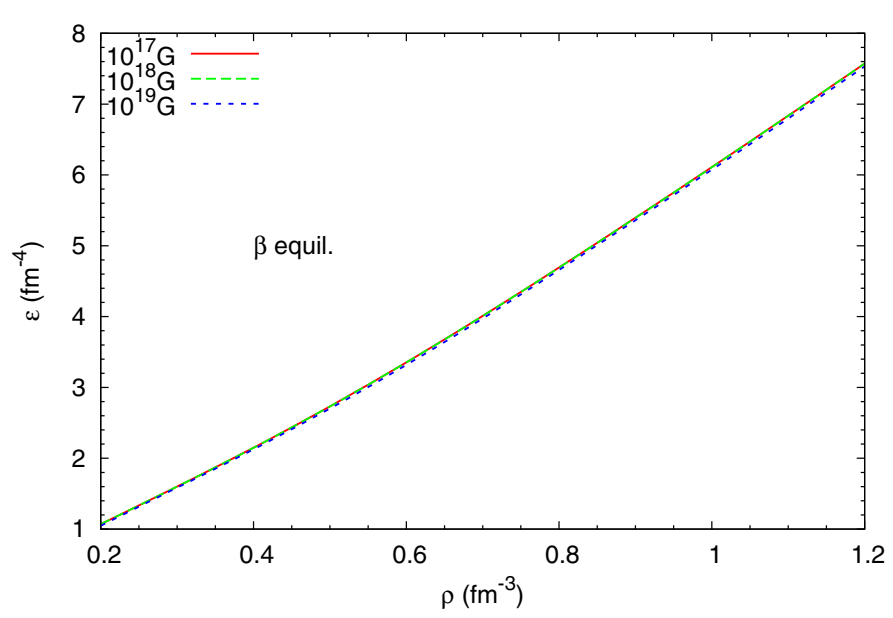

(a)

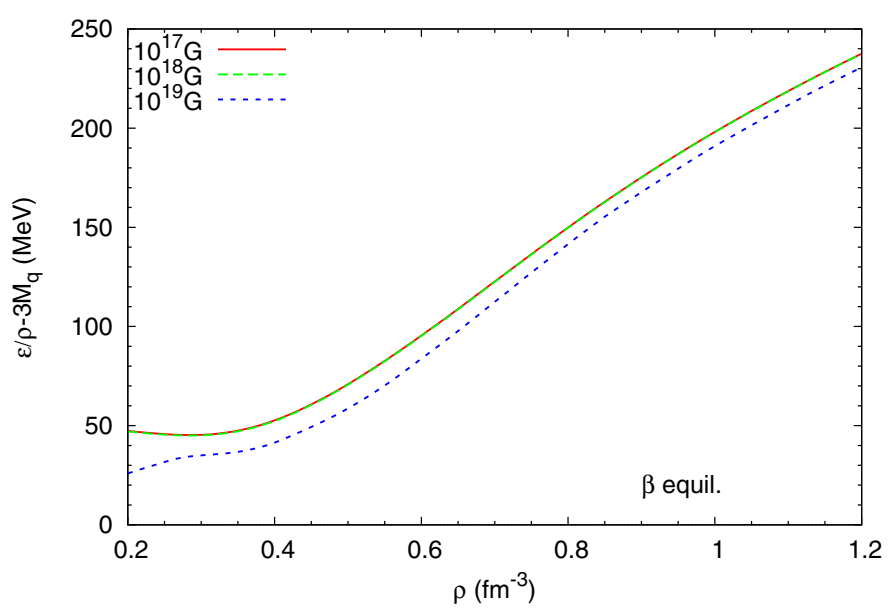

(c)

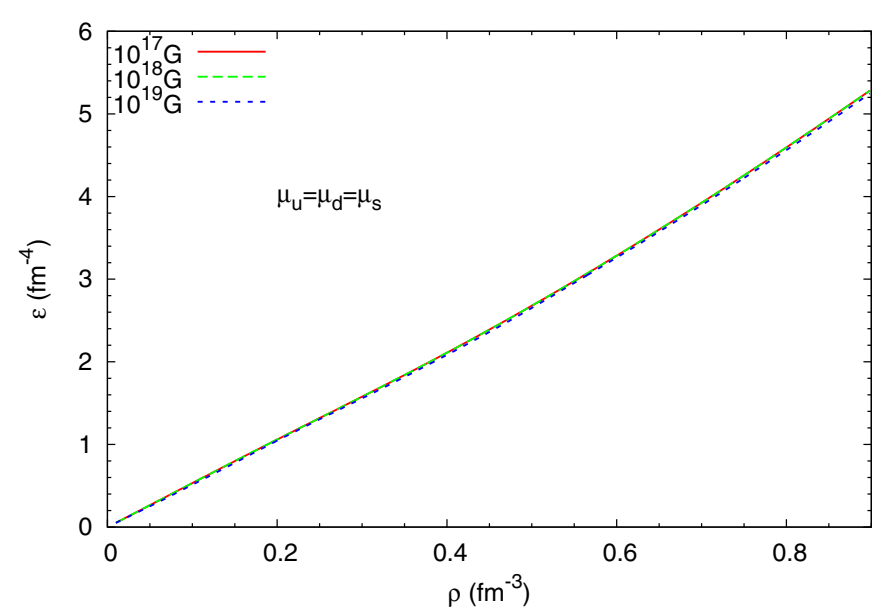

(b)

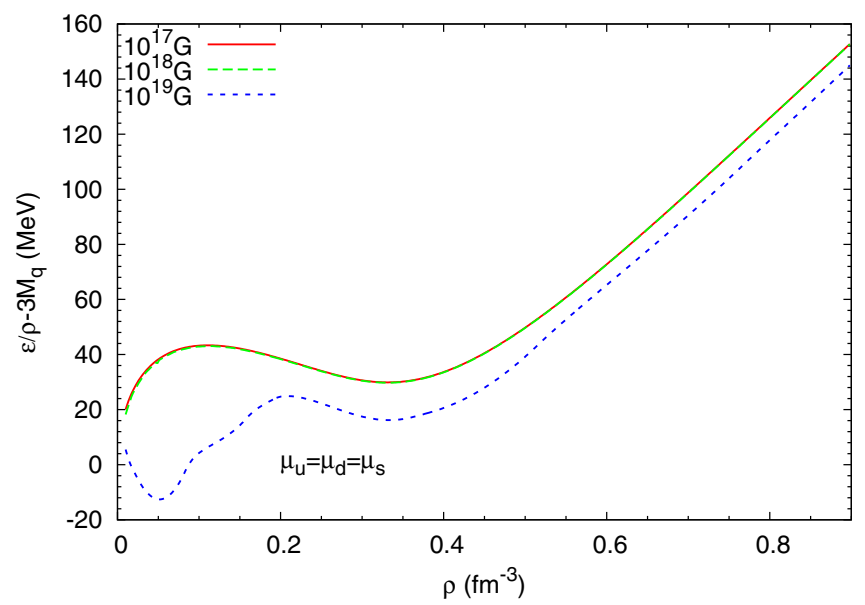

(d)

FIG. 4. (Color online) (a) and (b) Energy density and (c) and (d) quark binding energy for matter in $\beta$-equilibrium in the left panels and symmetric matter in the right panels.

as the MIT bag model, or a model without the strangeness degree of freedom, such as the su(2) NJL, is used to describe $\beta$-equilibrium quark matter, a different behavior will probably occur for $B \leqslant 10^{18} \mathrm{G}$ and $\rho>0.5 \mathrm{fm}^{-3}$ right until the star center, e.g., $\rho \sim 1.2 \mathrm{fm}^{-3}$ [21,55]; in particular, both pressure contributions will be coincident.

Therefore within the su(3) NJL model, a non-negligible effect of the magnetic field on the quark star structure close to the surface and in the interior is expected even for $B \sim 10^{18} \mathrm{G}$. Taking as reference the calculation done in Ref. [57] using pure toroidal magnetic field equilibrium models of relativistic stars for both nonrotating and rotating hadronic stars, fields as large as $10^{18} \mathrm{G}$ were obtained on the equatorial plane deep inside the star. In this calculation, however, the magnetic field effects on the EOS have been neglected.

We have then looked at the pressure anisotropy obtained from the components of the energy-momentum tensor that define the parallel and the perpendicular pressure contributions and examined the relation between parallel and perpendicular pressures for both scenarios described above when the magnetic field is fixed and also when the baryonic density is kept constant. We have observed that as the magnetic field intensity is larger, the discontinuities in the perpendicular pressure are also larger. It is important to note that the magnetization is not responsible for the complete picture, because it comes multiplied by the magnetic field in the calculation of the perpendicular pressure. In agreement with Refs. [27,31] we have observed that when the densities are fixed, the parallel and perpendicular pressures are practically coincident up to very large magnetic fields, what could justify the use of isotropic matter hydrostatic equations in stellar calculations. However, the integration of the full relativistic hydrostatic equations still requires the inclusion of the magnetic field contributionss which involve a $B^{2}$ term that gives rise to a quite large effect.

At this point, we would like to emphasize that compact star macroscopic properties, as masses and radii, were not computed because to trust our results we would need either a self-consistent model, as the one developed in Refs. [43-45], or a simpler approach, as the one in Ref. [40] that uses a more appropriate space geometry. In Ref. [45] it was shown that the contribution to the EOS of magnetic fields of the order of $7 \times 10^{17} \mathrm{G}$ is negligible on the determination of the maximum mass compared to the uncertainties existing among the EOS models. However, if we consider that a quark star is self-bound, 
we may expect that magnetic fields $\sim 10^{19} \mathrm{G}$ or larger exist in stable stars [25,49], and, in this case, the effects of magnetic field on the EOS (see Fig. 3) are probably non-negligible on the determination of the star structure.

One should also notice that strong magnetic fields affect the proton and electron fractions of $\beta$-equilibrium stellar matter. In particular it was shown that if the electrons and protons are confined to their LLL, the Urca reactions are open for an arbitrary proton concentration leading to a fast neutron star cooling [58], because the transversal momentum of charged particles is defined only within an accuracy of $\Delta p_{\perp} \sim \sqrt{e B}$. If this quantity is of the order of the neutron Fermi momentum direct Urca processes are allowed. In Ref. [59] is was discussed that even for much weaker fields there is already a noticeable effect corresponding to a speeding up of the cooling near the center of the star. Recently, it was also shown that neutrino emissivity will be enhanced due to $B$-induced unpairing of proton condensates [60]. With quark matter the situation is different. Unpaired quark matter may speed up the star cooling if the pairing nucleon gap is large [61], but color superconducting quark matter hinders neutrino emission. However, if unpaired quark matter is considered under the effect of strong magnetic fields it has been discussed that neutrino emission may also be hindered because direct Urca will only occur under very specific conditions [62]. This effect requires that all particles are in their LLL. In the present calculation we have seen that for $B \lesssim 10^{19} \mathrm{G}$ this is not the case, and therefore, within NJL the quark direct Urca process will not be hindered for realistic magnetic field intensities.

Finally, we point out that other physical aspects, such as the fermion anomalous magnetic moment, may also be important for a more realistic description when fields in excess of $10^{19} \mathrm{G}$ are considered. Indeed, the introduction of a magnetic field induces a new term corresponding to the coupling between the field and the fermion anomalous magnetic moment (AMM) which may influence the EoS for very high values of $B$. In Ref. [63] it has been shown that the inclusion of the anomalous magnetic moment contribution stiffens the stellar matter EOS if $B>10^{18} \mathrm{G}$, and may originate a total spin polarization of neutrons. For magnetic field intensities below the critical intensity, obtained equating the particle cyclotron energy to its rest mass, the Schwinger perturbative determination of the anomalous magnetic moment, corresponding to the lowest order correction to the magnetic moment obtained at tree level, is valid. Recently, it has been shown that for quark matter the scale for the perturbative approach is set by the constituent quark mass [64], and therefore, the effect of including an AMM should also be considered when describing quark matter under the effect of strong magnetic fields. With a mass about one third the nucleon mass, the critical field will be approximately one order of magnitude smaller the nucleon critical field, but still larger than the maximum field expected inside a neutron or quark star. Taking into account the AMM will certainly lift the degeneracy of Landau levels.

\section{ACKNOWLEDGMENTS}

D.P.M. and M.B.P. are partially supported by Conselho Nacional de Desenvolvimento Científico e Tecnológico (CNPqBrazil) and by Fundação de Amparo à Pesquisa e Inovação do Estado de Santa Catarina (FAPESC-Brazil), under Project No. 2716/2012. C.P. acknowledges financial support by Project No. PEst-OE/FIS/UI0405/2014 developed under the initiative QREN financed by the UE/FEDER through the program COMPETE "Programa Operacional Factores de Competitividade and by "NewCompstar", COST Action MP1304.

\section{APPENDIX: DERIVATIVES WITH RESPECT TO $B$}

As already shown in the text, the magnetization is given by

$$
\mathcal{M}=-\left(\frac{d \Omega}{d B}\right)_{\mu}=-\left(\frac{\partial \Omega}{\partial B}\right)_{\mu}-\sum_{f}\left(\frac{\partial \Omega}{\partial M_{f}}\right)_{\mu} \frac{d M_{f}}{d B},
$$

but in equilibrium

$$
\left(\frac{\partial \Omega}{\partial M_{f}}\right)_{\mu}=0
$$

and then

$$
M=-\left(\frac{\partial \Omega}{\partial B}\right)_{\mu}
$$

Thus, in the following expressions we do not need to include terms containing $\frac{d M_{f}}{d B}$ which also means that the vacuum contributions $\theta_{f}^{\prime \text { vac }}$ and $\phi_{f}^{\prime \text { vac }}$ trivially vanish. Then,

$$
\begin{aligned}
\theta_{f}^{\prime \mathrm{mag}}= & 2 \frac{\theta_{f}^{\mathrm{mag}}}{B}-\frac{N_{c}\left|q_{f}\right| B}{2 \pi^{2}} \frac{M_{f}^{2}}{2 B} \\
& \times\left[\ln \Gamma\left(x_{f}\right)-\frac{1}{2} \ln (2 \pi)+x_{f}-\left(x_{f}-\frac{1}{2}\right) \ln \left(x_{f}\right)\right],
\end{aligned}
$$

and

$$
\begin{aligned}
\theta_{f}^{\prime \text { med }}= & \frac{\theta_{f}^{\text {med }}}{B}-\frac{N_{c}\left|q_{f}\right| B}{4 \pi^{2}} \sum_{k=0}^{k_{f, \max }} \alpha_{k} k\left|q_{f}\right| \\
& \times \int_{-\infty}^{+\infty} d p \frac{1}{E_{f}^{*}}\left[f_{+}+f_{-}\right] .
\end{aligned}
$$

For $T=0$ the above relation becomes

$$
\begin{aligned}
\theta_{f}^{\prime \text { med }}= & \frac{\theta_{f}^{\text {med }}}{B}-\frac{N_{c} B\left|q_{f}\right|}{2 \pi^{2}} \sum_{k=0}^{k_{\max }} \alpha_{k} \\
& \times \ln \left(\frac{\mu_{f}+\sqrt{\mu_{f}^{2}-s_{f}^{2}}}{s_{f}}\right) k\left|q_{f}\right| .
\end{aligned}
$$

A straightforward evaluation yields

$$
\phi_{f}^{\prime \mathrm{mag}}=\frac{\phi_{f}^{\mathrm{mag}}}{B}+\frac{N_{c}}{4 \pi^{2}} \frac{M_{f}^{2}}{2 B}\left\{\left|q_{f}\right| B+M_{f}^{2}\left[\psi^{(0)}\left(x_{f}\right)-\ln \left(x_{f}\right)\right]\right\},
$$


where $\psi^{0}\left(x_{f}\right)=\frac{\Gamma^{\prime}\left(x_{f}\right)}{\Gamma\left(x_{f}\right)}$ is the $\digamma$ function. The in medium contribution reads

$$
\phi_{f}^{\prime \text { med }}=\frac{\phi_{f}^{\text {med }}}{B}-\frac{N_{c}\left|q_{f}\right| B}{4 \pi^{2}} \sum_{k=0}^{k_{f, \max }} \alpha_{k} k\left|q_{f}\right| \int_{-\infty}^{+\infty} d p\left\{\frac{f_{+}}{\left(E_{f}^{*}\right)^{2}}\left[\frac{1}{E_{f}^{*}}+\frac{f_{+}}{T} \exp \left[\left(E_{f}^{*}-\mu_{f}\right) / T\right]\right]+\left(\mu \leftrightarrow-\mu{ }^{\prime} f_{+} \leftrightarrow f_{-}\right)\right\},
$$

which, at $T=0$, can be written as

$$
\phi_{f}^{\prime \text { med }}=\frac{\phi_{f}^{\text {med }}}{B}-\frac{N_{c}\left|q_{f}\right| B}{2 \pi^{2}} \sum_{k=0}^{k_{f, \max }} \alpha_{k} \frac{\mu_{f} M_{f} k\left|q_{f}\right|}{s_{f}(k, B)^{2} \sqrt{\mu_{f}^{2}-s_{f}(k, B)^{2}}}
$$

[1] E. S. Fraga, arXiv:1310.6656 [hep-ph]; J. O. Andersen, W. R. Naylor, and A. Tranberg, arXiv:1411.7176 [hep-ph]; V. A. Miransky and I. A. Shovkovy, Phys. Rep. 576, 1 (2015).

[2] T. Inagaki, D. Kimura, and T. Murata, Prog. Theo. Phys. 111, 371 (2004).

[3] F. Preis, A. Rebhan, and A. Schmitt, J. High Energy Phys. 03 (2011) 033; Lect. Notes Phys. 871, 51 (2013).

[4] M. D'Elia, S. Mukherjee, and F. Sanfilippo, Phys. Rev. D 82, 051501 (2010).

[5] E.-M. Ilgenfritz, M. Kalinowski, M. Müller-Preussker, B. Petersson, and A. Schreiber, Phys. Rev. D 85, 114504 (2012).

[6] G. S. Bali, F. Bruckmann, G. Endrödi, Z. Fodor, S. D. Katz, S. Krieg, A. Schäfer, and K. K. Szabó, J. High Energy Phys. 02 (2012) 044.

[7] G. S. Bali, F. Bruckmann, G. Endrödi, Z. Fodor, S. D. Katz, and A. Schäfer, Phys. Rev. D 86, 071502(R) (2012).

[8] K. Fukushima and Y. Hidaka, Phys. Rev. Lett. 110, 031601 (2013); T. Kojo and N. Su, Phys. Lett. B 720, 192 (2013); F. Bruckmann, G. Endrodi, and T. G. Kovacs, J. High Energy Phys. 04 (2013) 112; E. S. Fraga, J. Noronha, and L. F. Palhares, Phys. Rev. D 87, 114014 (2013).

[9] M. Ferreira, P. Costa, D. P. Menezes, C. Providência, and N. N. Scoccola, Phys. Rev. D 89, 016002 (2014); M. Ferreira, P. Costa, O. Lourenço, T. Frederico, and C. Providência, ibid. 89, 116011 (2014); R. L. S. Farias, K. P. Gomes, G. I. Krein, and M. B. Pinto, Phys. Rev. C 90, 025203 (2014).

[10] S. S. Avancini, D. P. Menezes, M. B. Pinto, and C. Providência, Phys. Rev. D 85, 091901(R) (2012).

[11] P. Costa, M. Ferreira, H. Hansen, D. P. Menezes, and C. Providência, Phys. Rev. D 89, 056013 (2014).

[12] A. G. Grunfeld, D. P. Menezes, M. B. Pinto, and N. N. Scoccola, Phys. Rev. D 90, 044024 (2014).

[13] K. Fukushima, D. E. Kharzeev, and H. J. Warringa, Phys. Rev. D 78, 074033 (2008); D. E. Kharzeev and H. J. Warringa, ibid. 80, 034028 (2009); D. E. Kharzeev, Nucl. Phys. A 830, 543c (2009).

[14] R. Duncan and C. Thompson, Astrophys. J. 392, L9 (1992); C. Kouveliotou et al., Nature (London) 393, 235 (1998).

[15] K. Tuchin, Adv. High Energy Phys. 2013, 490495 (2013); Phys. Rev. C 88, 024911 (2013).

[16] L. McLerran and V. Skokov, Nucl. Phys. A 929, 184 (2014).

[17] A. Bzdak and V. Skokov, Phys. Lett. B 710, 171 (2012); W.-T. Deng and X.-G. Huang, Phys. Rev. C 85, 044907 (2012).

[18] M. G. de Paoli and D. P. Menezes, Adv. High Energy Phys. 2014, 479401 (2014)
[19] G. Basar, D. E. Kharzeev, and V. Skokov, Phys. Rev. Lett. 109, 202303 (2012); A. Bzdak and V. Skokov, ibid. 110, 192301 (2013).

[20] D. P. Menezes, M. B. Pinto, S. S. Avancini, A. P. Martínez, and C. Providência, Phys. Rev. C 79, 035807 (2009).

[21] D. P. Menezes, M. B. Pinto, S. S. Avancini, and C. Providência, Phys. Rev. C 80, 065805 (2009).

[22] L. L. Lopes and D. P. Menezes, Braz. J. Phys. 42, 428 (2012).

[23] R. H. Casali, L. B. Castro, and D. P. Menezes, Phys. Rev. C 89, 015805 (2014).

[24] R. D. Blandford and L. Hernquist, J. Phys. C 15, 6233 (1982).

[25] E. J. Ferrer, V. de la Incera, J. P. Keith, I. Portillo, and P. L. Springsteen, Phys. Rev. C 82, 065802 (2010).

[26] X.-G. Huang, M. Huang, D. H. Rischke, and A. Sedrakian, Phys. Rev. D 81, 045015 (2010).

[27] L. Paulucci, E. J. Ferrer, V. de la Incera, and J. E. Horvath, Phys. Rev. D 83, 043009 (2011).

[28] D. Manreza Paret, J. E. Horvath, and A. Perez Martinez, arXiv:1407.2280 [astro-ph.HE].

[29] G. S. Bali, F. Bruckmann, G. Endrodi, and A. Schaefer, PoS LATTICE 2013, 182 (2014).

[30] R. C. Tolman, Phys. Rev. 55, 364 (1939); J. R. Oppenheimer and G. M. Volkoff, ibid. 55, 374 (1939).

[31] V. Dexheimer, D. P. Menezes, and M. Strickland, J. Phys. G 41, 015203 (2014).

[32] D. Bandyopadhyay, S. Chakrabarty, and S. Pal, Phys. Rev. Lett. 79, 2176 (1997).

[33] G. J. Mao, C. J. Mao, A. Iwamoto, and Z. X. Li, Chin. J. Astron. Astrophys. 3, 359 (2003).

[34] A. Rabhi et al., J. Phys. G 36, 115204 (2009).

[35] C. Y. Ryu, K. S. Kim, and M.-K. Cheoun, Phys. Rev. C 82, 025804 (2010).

[36] A. Rabhi, P. K. Panda, and C. Providência, Phys. Rev. C 84, 035803 (2011).

[37] R. Mallick and M. Sinha, Mon. Not. R. Astron. Soc. 414, 2702 (2011).

[38] V. Dexheimer, R. Negreiros, and S. Schramm, Eur. J. Phys. A 48, 189 (2012).

[39] D. P. Menezes, M. B. Pinto, L. B. Castro, P. Costa, and C. Providência, Phys. Rev. C 89, 055207 (2014).

[40] R. Mallick and S. Schramm, Phys. Rev. C 89, 045805 (2014).

[41] R. O. Gomes, V. Dexheimer, and C. A. Z. Vasconcellos, Astron. Nachr. 335, 666 (2014). 
[42] L. L. Lopes and D. P. Menezes, arXiv:1411.7209.

[43] M. Bocquet et al., Astron. Astrophys. 301, 757 (1995).

[44] C. Y. Cardall, M. Prakash, and J. M. Lattimer, Astrophys. J. 554, 322 (2001).

[45] D. Chatterjee, T. Elghozi, J. Novak, and M. Oertel, arXiv: $1410.6332 \mathrm{v} 1$ [astro-ph.HE].

[46] C. W. Misner, K. S. Thorne, and J. A. Wheeler, Gravitation (Freeman and Company, San Francisco, 1973).

[47] Ya. B. Zel'dovich and I. D. Nivikov, Stars and Relativity (Dover, New York, 1996).

[48] A. Broderick, M. Prakash, and J. M. Lattimer, Phys. Lett. B 531, 167 (2002).

[49] J. L. Noronha and I. A. Shovkovy, Phys. Rev. D 76, 105030 (2007).

[50] Y. Nambu and G. Jona-Lasinio, Phys. Rev. 122, 345 (1961); 124, 246 (1961).

[51] M. Buballa, Phys. Rep. 407, 205 (2005).

[52] T. Hatsuda and T. Kunihiro, Phys. Rep. 247, 221 (1994).

[53] R. G. Felipe, A. P. Martinez, H. P. Rojas, and M. Orsaria, Phys. Rev. C 77, 015807 (2008).
[54] Ch. Kettner, F. Weber, M. K. Weigel, and N. K. Glendenning, Phys. Rev. D 51, 1440 (1995).

[55] D. P. Menezes, C. Providência, and D. B. Melrose, J. Phys. G 32, 1081 (2006).

[56] S. S. Avancini, D. P. Menezes, and C. Providência, Phys. Rev. C 83, 065805 (2011).

[57] J. Frieben and L. Rezzolla, MNRAS 427, 3406 (2012).

[58] L. B. Leinson and A. Perez, J. High Energy Phys. 09 (1998) 020.

[59] D. A. Baiko and D. G. Yakovlev, Astron. Astrophys. 342, 192 (1999).

[60] M. Sinha and A. Sedrakian, Phys. Rev. C 91, 035805 (2015).

[61] D. Page, M. Prakash, J. M. Lattimer, and A. W. Steiner, Phys. Rev. Lett. 85, 2048 (2000); D. Page, J. M. Lattimer, M. Prakash, and A. W. Steinerand, Astrophys. J., Suppl. Ser. 155, 623 (2004).

[62] C. Kouvaris, Phys. Rev. D 79, 123008 (2009).

[63] A. Broderick, M. Prakash, and J. M. Lattimer, Astrophys. J. 537, 351 (2000).

[64] L. Chang, Y.-X. Liu, and C. D. Roberts, Phys. Rev. Lett. 106, 072001 (2011). 